\title{
A novel approach to the transient ventilation of road tunnels
}

\author{
Hong-Ming Jang ${ }^{\mathrm{a}}$, Falin Chen ${ }^{\mathrm{b}, *}$
}

${ }^{a}$ Department of Mechanical Engineering, Chinese Culture University,Taipei, Taiwan 111, Republic of China

${ }^{\mathrm{b}}$ Institute of Applied Mechanics, National Taiwan University, Taipei, Taiwan 107, Republic of China

Received 25 April 1999; received in revised form 25 August 1999; accepted 25 October 1999

\begin{abstract}
We propose in this paper a novel approach to predict the transient behavior of the ventilation of road tunnel based on the traffic data measured at the outlet portal of tunnel. The approach starts with a so-called traffic group-partition dilemma, which essentially partitions the traffic flow into various groups so that the transient behavior of the induced wind speed can be accurately predicted. As a result, one may employ the present approach to predict the ventilation situation along a road tunnel, which can be a long tunnel in motorway, and needs only to measure the traffic data at one location in or near the tunnel, for example, the outlet portal. Accordingly, the present approach shall become a rather economic and efficient scheme to be considered to monitor the ventilation situation in road tunnels as the approach is converted into a computer program, which can be used in the real-time ventilation control of the tunnel (C) 2000 Elsevier Science Ltd. All rights reserved.
\end{abstract}

Keywords: Ventilation; Tunnel

\section{Introduction}

A well-designed ventilation system of road tunnel ensures not only good air quality but also safety for the users of tunnel. Engineers are constantly searching for an economic and reliable way of ventilation, which can efficiently exchange the air between atmosphere and tunnel. For a tunnel with one-bound traffic, the piston effect due to moving vehicles is the major factor driving the motion of the air in tunnel. The

\footnotetext{
*Corresponding author. Fax: + 886-2-36-39290.
} 
mechanical devices, such as jet fans, serve only when an emergency state is alarmed. The wind speed induced by the piston effect accordingly becomes a major issue to be studied when the design of ventilation proceeds. To simulate the wind induced by traffic, the one-dimensional mathematical model based on the conservation of momentum within the tunnel is usually considered [1-4]. It is noted that the transient state of ventilation is crucial to the users of tunnel, in terms of both the quality of air and the safety during emergency. We therefore in the present paper propose a novel approach to predict the transient behavior of the ventilation in one-bound traffic tunnel.

To study the transient behavior of ventilation in road tunnel, one may investigate the unsteady flow field within the tunnel. We nevertheless note that to study the unsteady two- or three-dimensional flow in road tunnel, especially for which is of several kilometers long, the approach through numerical computation is rather inefficient and non-practical. Our present approach is developed upon the basis of one-dimensional model. It is noted that the factors influencing the fluid motion in tunnel include the motion of vehicles, the pressure rise imposed by the jet fans, the pressure difference between inlet and outlet portals, the resistance due to tunnel wall, and so on [4]. Among these factors the dynamic variation of the traffic flow and the switch-on and -off of the jet fan are dominant [5]. In a road tunnel, the traffic flow changes rapidly and thus the induced wind speed shall be of significant variation with time. In order to reveal the intimate relation between the transient traffic and the fluctuating wind speed, we must first obtain the real transient traffic information along the tunnel and then properly introduce it into the theoretical model [5]. To reach this goal, nevertheless, collecting the complete vehicular traffic information along the tunnel is by no means practical. The practical way is instead to measure the traffic flow at a particular location in or near the tunnel, which can be the outlet portal, for example. The traffic flow in terms of the traffic density as well as the speed of vehicles are then analyzed on the basis of the so-called traffic group-partition dilemma, with which the traffic flow is partitioned into vehicle-groups. Consequently, the transient behavior of the ventilation in any location of the whole tunnel, in terms of the time variations of wind speed, pressure, and temperature, can all be predicted by the theoretical approach based on the traffic data measured at one location. When the present approach is converted into computer code, the real time control of the ventilation of the whole tunnel can be made possible if only one point of traffic data is provided. The present approach may therefore provide an efficient and economic way for the ventilation control in road tunnel.

In the following paper, we will first in Section 2 describe the Fu-De tunnel in which the experiments were implemented. In Section 3 the typical traffic flow and the ventilation situation of $\mathrm{Fu}-\mathrm{De}$ tunnel are illustrated. The 1-D theoretical model of the ventilation is derived in Section 4. The so-called traffic group-partition dilemma is described in Section 5, in which the procedure and rules prescribed for the partition process are discussed. In Section 6 an intensive comparison between the computed results and the corresponding measured data is carried out. Finally, conclusions reached on the basis of present analyses are given in section 7 . 


\section{Description of tested tunnel and experimental facilities}

The tunnel tested is the Fu-De tunnel of the Northern Second Motorway (NSM) of Taiwan, locating in the suburban of Taipei city, the capital of Taiwan having a population about 2.6 million. It is one of the several long tunnels of the NSM, consisting of two one-bound-traffic tubes, one south bound $(1762 \mathrm{~m})$ and one north bound (1726m), each of which has three lanes, see Fig. 1(a) in which the outlet portal is shown. All the testing facilities were setup in the southbound tube. From Fig. 1(a) one can see that the traffic was heavy while was still smooth. Up to the present days, most of the vehicles using $\mathrm{Fu}$-De tunnel have been the small-size passenger cars. The traffic density of large-sized truck or trailer is rather low. On the upper-right corner of Fig. 1(a) shows a CCD camera which is used by the Motorway Engineering Bureau to monitor the traffic of tunnel, not the one used by us to measure the traffic data. The weather was rainy and foggy, not unusual in the summer of Taipei.

We set up the following testing facilities along the $\mathrm{Fu}$-De tunnel. On the righthand- side of the outlet portal, two sets of data acquisition system were equipped, see Fig. 1(b). The upper-larger box contained the data logger to collect the data of wind speed, pressure, temperature, humidity, and the concentration of carbon monoxide (CO). The lower smaller box contained the data logger with which the traffic data detected by the computerized optical camera (called Autoscope) were recorded. All the data were temporarily recorded in the memory unit of the loggers and would be copied to the hard disk of a note book computer after a certain period. The data were then brought back to the laboratory to be analyzed. The time interval to record one set of data can be adjusted to be as small as $10 \mathrm{~s}$. The memory of the loggers was big enough that, based on this smallest time interval, a test period of more than $24 \mathrm{~h}$ could be proceeded.

The Autoscope was set up on the top of the tunnel outlet, facing down the outgoing traffic, see Fig. 1(c). In the same picture one can see the anemometer measuring wind velocity (including magnitude and direction) and the sensors measuring the temperature, pressure, and humidity of the atmosphere. The Autoscope system, including camera and data logger, was manufactured by the Image Sensing Systems Inc. and of model number 2004. Through the Autoscope the number of vehicles, speed of vehicles, and types of vehicles in both day and nighttime can be measured. We calibrated its accuracy regarding the number of vehicles by using a mechanical counter. After several times of calibration, each time a 30-min test was implemented, the error resulted from the Autoscope was found to be as low as 5\% or less.

The anemometer was a propeller-type sensor (Fig. 1(d)) to measure the wind velocity, manufactured by R. M. Young Co. of model 5103. The measurable range of wind speed was from 0 to $60 \mathrm{~m} / \mathrm{s}$ with an accuracy of $0.3 \mathrm{~m} / \mathrm{s}$. We noted that the induced wind in tunnel was essentially unidirectional and the wind speed varied in a range from 0 to $10 \mathrm{~m} / \mathrm{s}$, so that this type of anemometer was good for the test. The measurement was also made for the variations of pressure, temperature, $\mathrm{CO}$ concentration, and humidity although these measurements were primarily not to be used for the present analyses. They nevertheless can be good references for the engineers who are in charge of the supervision of both the traffic and the ventilation of the tunnel. 

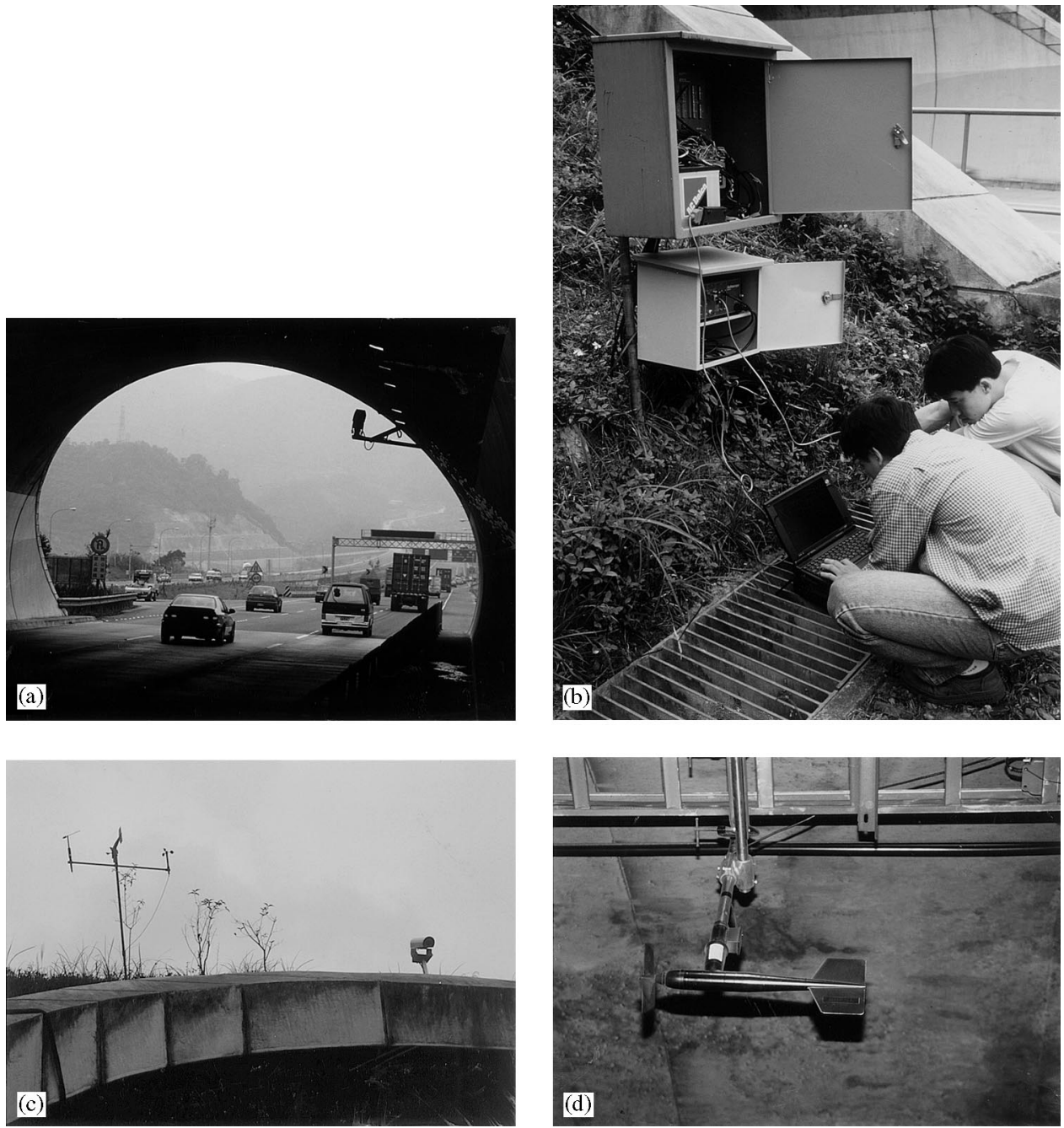

Fig. 1. The Fu-De tunnel and the testing facilities. (a) The outlet portal of the Fu-De tunnel. The tunnel has three lanes. The small-sized cars dominate the traffic flow. The CCD camera on the upper-right of tunnel is used by the Motorway Engineering Bureau to monitor the traffic of the tunnel. (b) The two data loggers for collecting the data of wind velocity, pressure, temperature, and so on (upper box) and for recording the data from the Autoscope camera (lower box). The two fellow students were transferring the data from both loggers to the hard disc of a notebook PC. (c) The Autoscope camera and the testing facility of the atmosphere data, including the wind speed and direction, temperature, pressure, humidity, and so on. (d) The anemometer in tunnel. The box shown on the upper-right corner of the picture contains the sensors for temperature, pressure, $\mathrm{CO}$ concentration, and humidity.

Along the southbound tube we measured three points, locating, respectively, at 50, 500 , and $1000 \mathrm{~m}$ from the outlet portal. On each point all the sensors mentioned above were set up. The sensors were fixed on the cable-truss, which was about $1 \mathrm{~m}$ below the ceiling of the tunnel. As we have found from the test results, the wind speed 
measured at these three locations were virtually the same, suggesting that the incompressible assumption is appropriate for the ventilation of road tunnel. The data regarding the wind speed will be used to test the accuracy of the present theoretical approach.

\section{Typical traffic flow and ventilation situation}

Figs. 2 and 3 illustrate the traffic flow on 17/02/97 (the 17th of February 1997), revealing the typical vehicular traffic on weekdays. In Fig. 2 the number of vehicle was recorded every $5 \mathrm{~min}$ (or called the traffic volume, defined as number of vehicles per unit time) and the data are shown on the basis of both each lane and of the three lanes as a whole. It is shown that the middle lane was always the first choice for a larger part of the drivers; the outer lane was the next and the inner lane was the last. The traffic was low from mid-night to about $6: 00 \mathrm{am}$, the number of vehicles in average was less than 20 vehicles per $5 \mathrm{~min}$. After $6: 00$ am the number of vehicles increased dramatically and reached the maximum at about $7: 30 \mathrm{am}$, and then remained in a heavytraffic situation to about 9:00 am and decreased afterwards. The heaviest traffic flow could be as high as 240 vehicles per $5 \mathrm{~min}$, being equivalent to a traffic density of $32 \mathrm{veh} / \mathrm{km}$, or about 55 vehicles in the southbound of $\mathrm{Fu}$-De tunnel at the same time. Starting from 10:00 am to $5: 00 \mathrm{pm}$, the averaged traffic volume was about 100 vehicles $5 \mathrm{~min}$. Another traffic peak occurred at about $6: 00 \mathrm{pm}$, the traffic volume

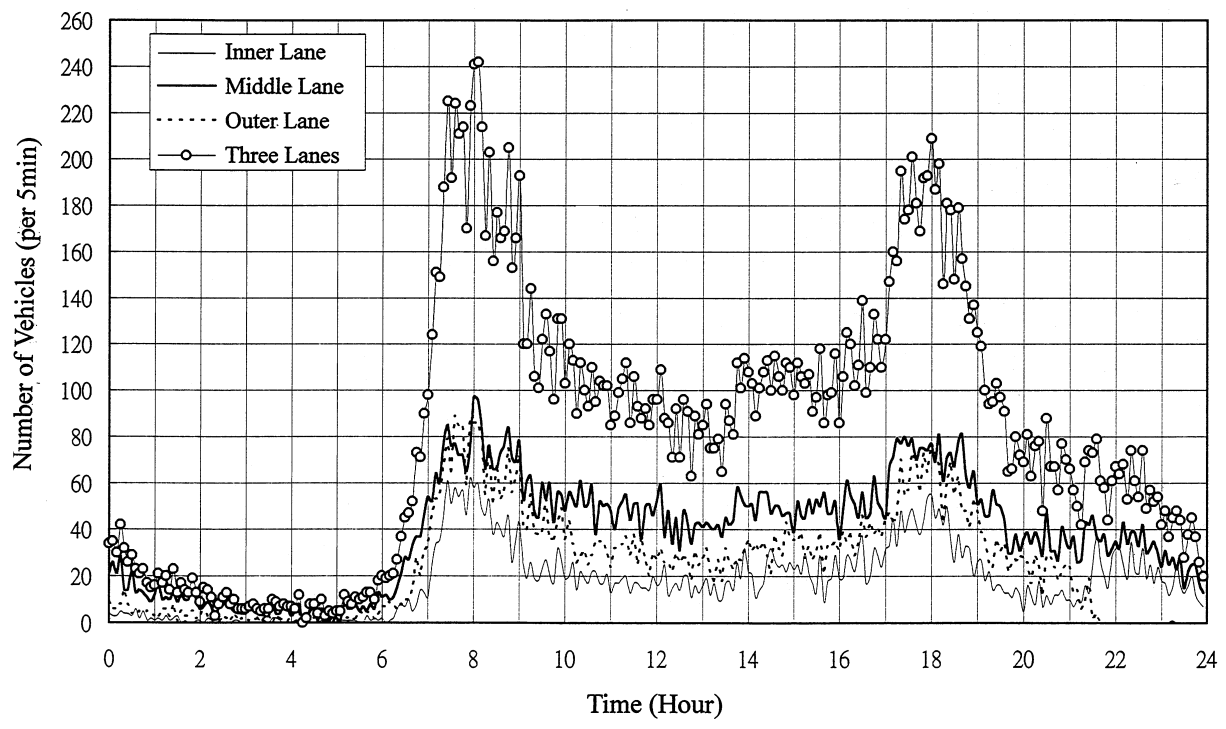

Fig. 2. The traffic flow accounted for by the number of vehicles passing through the outlet portal per $5 \mathrm{~min}$ on 17/02/97. Four sets of data represent, respectively, the number of vehicles in the outer lane, the inner lane, the middle lane, and the total number of the three lanes. It is seen that there were two peaks of the traffic flow, at, respectively, around $8: 00$ am and $6: 00 \mathrm{pm}$. 


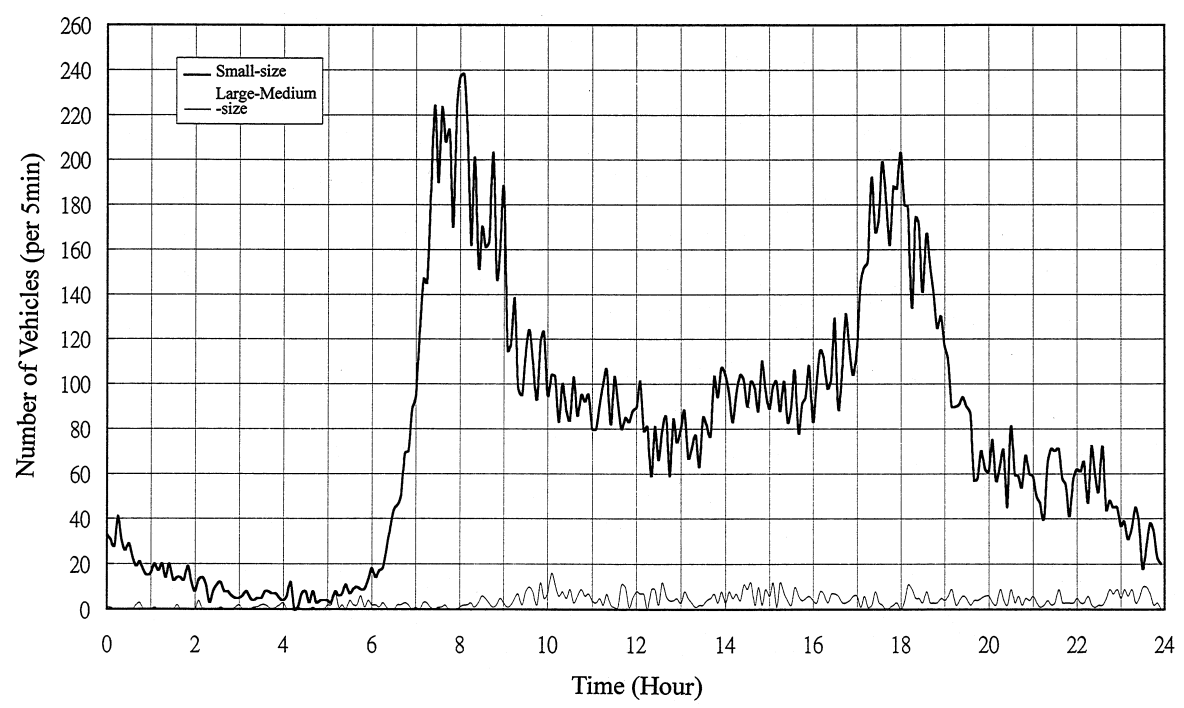

Fig. 3. The traffic flow accounted for by the number of vehicles passing through the outlet portal per $5 \mathrm{~min}$ on 17/02/97. Two sets of data represent, respectively, the traffic flow of the small-sized vehicles and the large- and medium-sized vehicles. It is seen that the small-sized car accounted for a great portion of the traffic.

was about 200 vehicles $5 \mathrm{~min}$, a little less than the peak at $8: 00 \mathrm{am}$. The traffic became more relax after $6: 00 \mathrm{pm}$, the traffic volume decreased gradually with time and reached to the minimum at around $4: 00 \mathrm{am}$.

Fig. 3 shows the traffic data of the same day, while the data were partitioned into two groups: the small-sized vehicles and large- and medium-sized vehicles. It is seen that more than $90 \%$ of the vehicles passing through $\mathrm{Fu}$-De tunnel were of small-sized vehicles. The large- and medium-sized vehicles showed up mostly in the daytime. We also show in Fig. 4(a) the speed of vehicle, which in average was about $90 \mathrm{~km} / \mathrm{h}$, just on the speed limit of motorway in Taiwan, suggesting that the traffic was smooth and normal and followed the criteria of design quite well. The variation of vehicle speed was quite dramatic during the night, which was because the traffic density was low and the averaged speed was evaluated on the basis of fewer vehicles. Fig. 4(b) illustrates the variation of the traffic density in the same day, showing that on the traffic peak the traffic density could be as high as $50 \mathrm{veh} / \mathrm{km}$ and the averaged value in the daytime was about $20 \mathrm{veh} / \mathrm{km}$.

It is interesting to see the variation of the wind speed, which is primarily induced by the motion of vehicles and accordingly is closely related to the traffic flow in tunnel. We show in Fig. 4(c) the variation of the wind speed measured at the point $50 \mathrm{~m}$ from the outlet portal. It is seen that the wind speed could be as high as $6 \mathrm{~m} / \mathrm{s}$ at the traffic peak, and was about $4 \mathrm{~m} / \mathrm{s}$ in the daytime and about $2 \mathrm{~m} / \mathrm{s}$ between $3: 00$ and 5:00 am. Obviously the wind speed increased with larger traffic flow, while it was not linearly proportional to the number of vehicles. Fig. 4(d) also shows the variation of the $\mathrm{CO}$ concentration, which again appeared similarly with the traffic flow density, 

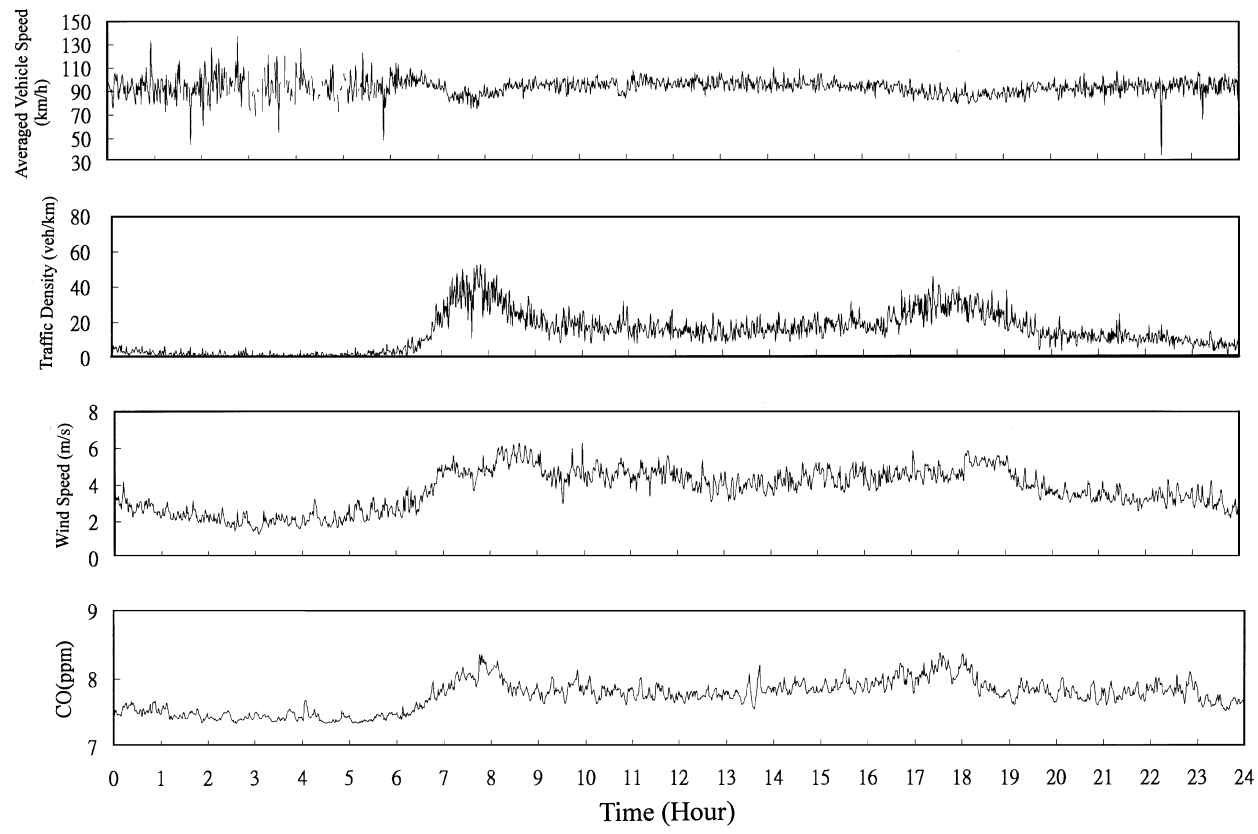

Fig. 4. Typical traffic flow and ventilation situation in Fu-De tunnel on 05/03/97. (a) Averaged vehicle speed $(\mathrm{km} / \mathrm{h})$. (b) Traffic density $(\mathrm{veh} / \mathrm{km})$. (c) Induced wind speed $(\mathrm{m} / \mathrm{s})$. (d) CO concentration $(\mathrm{ppm})$.

suggesting that the variation of the $\mathrm{CO}$ concentration be also closely related to the traffic flow.

\section{The one-dimensional theoretical model}

The induced wind speed in tunnel is mainly driven by both the piston force due to moving vehicles and the pressure rise imposed by jet fans. Besides, the friction of tunnel wall, the pressure difference between two portals, and the friction loss at the inlet of the tunnel also influence the wind speed although to a smaller extent. Based on the force balance among these factors, the one-dimensional force equation can be formulated in a control volume which is chosen to be confined by the tunnel wall, the cross section surfaces at the inlet and the outlet. The external forces imposing upon the control volume are the piston force due to vehicle motion $F_{1}$, the thrust force imposed by jet fans $F_{2}$, the force due to the static pressure difference between two portals $F_{3}$, the frictional resistance of tunnel wall $F_{4}$, and the frictional loss due to the flow separation at inlet portal $F_{5}$. The effect of the compressibility of air in the control volume is neglected. The relation between the resultant force and the induced wind speed $V$ can be written as

$$
\sum_{i=1}^{5} F_{i}=\rho A_{\mathrm{t}} L \frac{\mathrm{d} V}{\mathrm{~d} t},
$$


where $F_{i}, i=1,5$ are

$$
\begin{aligned}
F_{1} & =\sum_{j=1}^{N} \frac{\rho}{2} C d_{j}\left(U_{j}-V\right)\left|U_{j}-V\right| A v_{j}, \\
F_{2} & =n_{\mathrm{F}} \rho A_{\mathrm{F}}\left|V_{\mathrm{F}}\right|\left(V_{F}-V\right) K_{j}, \\
F_{3} & =\left(P_{\mathrm{i}}-P_{\mathrm{e}}\right) A_{\mathrm{t}}, \\
F_{4} & =-f \frac{\rho}{2} \frac{L}{D_{\mathrm{h}}} A_{\mathrm{t}} V|V|, \\
F_{5} & =-K_{\text {port }} \frac{\rho}{2} A_{\mathrm{t}} V|V|,
\end{aligned}
$$

In above equations, $\rho$ is the density of air, $A_{\mathrm{t}}$ is the mean cross section area of tunnel, $L$ is the length of tunnel, $C d_{j}, U_{j}$, and $A v_{j}$ are respectively the drag coefficient, the speed and the frontal area of vehicle $j, N$ is the total number of vehicles in tunnel, $f$ is the friction factor of tunnel wall, $n_{\mathrm{F}}, A_{\mathrm{F}}, V_{\mathrm{F}}, K_{j}$ are, respectively, the number, the discharging area, the discharging speed and the pressure-rise coefficient of jet fans, and $K_{\text {port }}$ is the friction loss coefficient of inlet portal. We also note that $P_{\mathrm{i}}, P_{\mathrm{e}}$ and $P_{\infty}$ are, respectively, the static pressures at inlet portal, outlet portal and atmosphere, which are related by

$$
P_{\mathrm{i}}=P_{\infty}-\frac{1}{2} \rho V^{2}-\rho \sqrt{\frac{A_{\mathrm{t}}}{2\left(\pi-\theta_{\mathrm{h}}\right)}} \frac{\mathrm{d} V}{\mathrm{~d} t},
$$

where $\theta_{\mathrm{h}}$ is the elevation angle of foothill slope around the inlet portal and $P_{\mathrm{e}}=P_{\infty}$ is considered. Eq. (7) is derived from the unsteady Bernoullis equation, in which the second term of the right-hand side accounts for the dynamic pressure and the third term accounts for the unsteady flow contribution on the pressure difference between two portals.

\section{Traffic group-partition dilemma}

Eq. (1) illustrates that there are five major external forces influencing the induced wind speed, and the piston effect of vehicle motion plays a major role in determining the transient behavior of induced wind. In fact, we have found that the traffic flow in $\mathrm{Fu}-\mathrm{De}$ tunnel varied constantly, suggesting that the unsteady transient behavior of the induced wind could predominate over the steady state to influence on the ventilation in tunnel. In order to more precisely reflect the transient phenomena of the induced wind, the variation of traffic flow must be described in details for the $F_{1}$ of Eq. (2). To account for the traffic flow variation, we propose accordingly a traffic group-partition dilemma, with which the unsteady features of the traffic flow can be captured on the basis of the groups of vehicles in tunnel. The unsteady induced wind speed can accordingly be calculated through Eq. (1) with an $F_{1}$ (see Eq. (21)) which can really reflect effects due to traffic flow through the group-partition dilamma to be described in the next two sections. 


\subsection{Background and rules of partition}

Not like previous studies [1-4] which considered that all the $N$ vehicles inside the tunnel were of the same drag coefficient $C_{\mathrm{d}}$, same frontal area $A_{\mathrm{v}}$, and same averaged speed $\bar{U}(t)$, we consider in this study different values of above three physical parameters based on a group of vehicles. The vehicles are separated into three different types according to the length of vehicle $l_{\mathrm{v}}$ detected by the optical Autoscope. The large-sized vehicles were defined to be of a length $l_{\mathrm{v}}>11 \mathrm{~m}$, the small-sized vehicles were of a length $l_{\mathrm{v}}<7.6 \mathrm{~m}$, and the medium-sized vehicles were of a length in between. The Autoscope also detected both the speed of each vehicle passing the outlet portal and the number of vehicles passed in each of the consecutive time intervals. Based on these data the averaged speed for each type of vehicle in each time interval were calcualted. Namely, the vehicles were partitioned according to types and time intervals. Both the number of vehicles and the averaged speed of each partitioned group were then recorded in the data logger. For the present data logger, the time interval is adjustable between $10 \mathrm{~s}$ to $60 \mathrm{~min}$.

For convenience for following discussion, we itemize the rules with which the traffic flow is partitioned:

1. Vehicles are partitioned into three types according to their lengths.

2. Vehicles of the same type, which pass the outlet portal in the same time interval, are considered to be in the same group.

3. The vehicles in the same group are assumed to travel through tunnel with a constant speed, which is equal to their averaged speed measured at the outlet portal.

Please note that since each group moves in its own speed, the overtaking between different groups is preserved in the present approach.

\subsection{Procedure of group partition}

Consider the traffic flow to be analyzed is in a period of $T \mathrm{~h}$ and the time interval to record the traffic flow is $\Delta t \mathrm{~s}$. This period of $T \mathrm{~h}$ is uniformly divided into $M$ intervals, so that $M=T \times 3600 / \Delta t$. In each $\Delta t$, vehicles are further separated into three groups according to their individual lengths. The vehicles passed the outlet portal in this time period are therefore totally partitioned into $3 M$ groups, whereas some of the groups may include zero vehicle. The group of type $i$ ( $i=1$ for small-sized vehicles, two for medium-sized vehicles, and three for large-sized vehicles) passing the outlet portal at the $k$ th time interval $(k=1,2,3, \ldots, M)$ is denoted by the subscript $i k$. The number and the averaged speed of the $i k$ th group are denoted by $n_{i k}$ and $\bar{U}_{i k}(\mathrm{~m} / \mathrm{s})$, respectively, which were measured by the Autoscope. The extent (or the length) covering the $i k$ th group is equal to the distance traveled by this group in the corresponding time interval, being denoted by $l_{\mathrm{i} k}$ and can be written as

$$
l_{i k}(m)=\bar{U}_{i k}(\mathrm{~m} / \mathrm{s}) \times \Delta t(\mathrm{~s}) .
$$

The leading position of this group $x_{\mathrm{i} k}(t)$ can be written as

$$
x_{i k}(t)=L+l_{i k} \frac{t-(k-1) \Delta t}{\Delta t} \quad(k-1) \Delta t \leqslant t \leqslant k \Delta t,
$$


where $L(\mathrm{~m})$ is the length of tunnel and the origin of the $x$ - coordinate is set to be at the inlet portal. Because the vehicles in the same group are assumed to travel with the same speed, the $l_{i k}$ must therefore be constant. The vehicles in the same group are assumed to be uniformly distributed so that the traffic density of this group is defined as $D_{i k}=n_{i k} / l_{i k}$, which accounts for the traffic density of vehicles of type $i$ in the region between $x=x_{i k}(t)-l_{i k}$ and $x=x_{i k}(t)$ at the moment $t$.

Consider first the case $l_{i k}<L$, all vehicles of the $\mathrm{i} k$ th group are assumed to be in tunnel at the beginning of the $k$ th time interval and the last vehicle of this group is assumed leaving tunnel at the end of this time interval. So that at any moment $t$ in this time interval, the number of vehicles of this group remaining in tunnel can be calculated by the following equation:

$$
n_{i k}^{\text {in }}(t)=n_{i k}-n_{i k} \frac{t-(k-1) \Delta t}{\Delta t} \quad(k-1) \Delta t \leqslant t \leqslant k \Delta t,
$$

where the superscript "in" means in tunnel. When $l_{i k}>L$ is considered, there are only $n_{i k} L / l_{i k}$ vehicles of the $i k$ th group travelling inside the tunnel at the beginning of the $k$ th time interval, and the rest are still not yet entering the inlet portal. In the next $\left(l_{i k}-L\right) / \bar{U}_{i k}$ s, the number of vehicles leaving the tunnel is equal to that entering it so that the number of vehicles of this group travelling in tunnel remains to be

$$
n_{i k}^{\text {in }}(t)=n_{i k} \frac{L}{l_{i k}} \quad(k-1) \Delta t \leqslant t \leqslant(k-1) \Delta t+\frac{l_{i k}-L}{\bar{U}_{i k}} .
$$

After that, the number of vehicles in tunnel of this $i k^{\text {th }}$ group reduces constantly with time, i.e.,

$$
n_{i k}^{\text {in }}(t)=n_{i k} \frac{k \Delta t-t}{\Delta t} \quad(k-1) \Delta t+\frac{l_{i k}-L}{\bar{U}_{i k}} \leqslant t \leqslant k \Delta t .
$$

Besides the three groups denoted by the subscript $i k$ for $i=1,2,3$, there probably also exist simultaneously in tunnel other groups which shall be passing the outlet portal in the next time intervals (i.e., $(k+1)$ th, $(k+2)$ th, etc.). To gain a complete traffic information along the tunnel for the $k$ th time interval, all of these groups must be taken into account. The number of vehicles and the averaged speed of the $i(k+1)$ th group are denoted, respectively, by $n_{i(k+1)}$ and $\bar{U}_{i(k+1)}$. The extent and the traffic density of the $i(k+1)$ th group are, respectively, denoted by $l_{i(k+1)}$ and $D_{i(k+1)}$, which can be calculated, respectively, by the following equations:

$$
\begin{aligned}
& l_{i(k+1)}(m)=\bar{U}_{i(k+1)}(m / s) \times \Delta t(s), \\
& D_{i(k+1)}=n_{i(k+1)} / l_{i(k+1)} .
\end{aligned}
$$

The leading position of this group is at $x_{i(k+1)}$, calculated by

$$
x_{i(k+1)}(t)=L-l_{i(k+1)}\left(\frac{k \Delta t-t}{\Delta t}\right) \quad(k-1) \Delta t \leqslant t \leqslant k \Delta t .
$$


The traffic density and the averaged vehicle speed in the region between $x=x_{i(k+1)}(t)-l_{i(k+1)}$ and $x=x_{i(k+1)}(t)$ of the $i(k+1)$ th group at moment $t$ are respectively, $D_{i(k+1)}$ and $\bar{U}_{i(k+1)}$.

When $l_{i(k+1)}<L / 2$ is considered, all of the vehicles of the $i(k+1)$ th group are already inside the tunnel at the beginning of the $k$ th time interval and its first vehicle shall reach the outlet portal at the end of this time interval. Let $n_{i(k+1)}^{\text {in }}(t)$ denote the number of vehicles belonging to the $i(k+1)$ th group, we can write it as

$$
n_{i(k+1)}^{\text {in }}(t)=n_{i(k+1)} \quad(k-1) \Delta t \leqslant t \leqslant k \Delta t .
$$

When $L / 2<l_{i(k+1)}<L$ is considered, there are only $\left(\left(L-l_{i(k+1)}\right) / l_{i(k+1)}\right) n_{i(k+1)}$ vehicles of the $i(k+1)$ th group travelling inside the tunnel at the beginning of the $k$ th time interval. In the next $\left(2 l_{i(k+1)}-L\right) / \bar{U}_{i(k+1)}$ s, more vehicles of this group shall be travelling into tunnel until finally all $n_{i(k+1)}$ vehicles of this group are in tunnel. The value of $n_{i(k+1)}^{\text {in }}$ is calculated by

$$
\begin{aligned}
& n_{i(k+1)}^{\text {in }}(t)=\left(\frac{L-l_{i(k+1)}}{l_{i(k+1)}}\right) n_{i(k+1)}+\frac{t-(k-1) \Delta t}{\Delta t} n_{i(k+1)}, \\
& (k-1) \Delta t \leqslant t \leqslant(k-1) \Delta t+\frac{2 l_{i(k+1)}-L}{\bar{U}_{i(k+1)}}, \\
& n_{i(k+1)}^{\text {in }}(t)=n_{i(k+1)} \quad(k-1) \Delta t+\frac{2 l_{i(k+1)}-L}{\bar{U}_{i(k+1)}} \leqslant t \leqslant k \Delta t .
\end{aligned}
$$

When $l_{i(k+1)}>L$, there is no vehicle of the $i(k+1)$ th group travelling in tunnel at the beginning of the $k$ th time interval. The first vehicle of this group will not enter the inlet portal until $t=(k-1) \Delta t+\left(l_{i(k+1)}-L\right) / \bar{U}_{i(k+1)}$ and then other vehicles of this group shall continuously enter the tunnel afterwards. At the end of this time interval the first vehicle of the group will reach the outlet portal while some vehicles of this group remain outside from the inlet portal. Then the number of vehicles of the $i(k+1)$ th group in tunnel during the $k$ th time interval can be written as

$$
\begin{aligned}
& n_{i(k+1)}^{\mathrm{in}}(t)=0 \quad(k-1) \Delta t \leqslant t \leqslant(k-1) \Delta t+\frac{l_{i(k+1)}-L}{\bar{U}_{i(k+1)}}, \\
& n_{i(k+1)}^{\mathrm{in}}(t)=\frac{t-\left[(k-1) \Delta t+\left(l_{i(k+1)}-L\right) / \bar{U}_{i(k+1)}\right]}{\Delta t} n_{i(k+1),} \\
& (k-1) \Delta t+\frac{l_{i(k+1)}-L}{\bar{U}_{i(k+1)}} \leqslant t \leqslant k \Delta t .
\end{aligned}
$$

In the similar manner we can also deduce those information, respectively, for the $i(k+2)$ th group, $i(k+3)$ th group, $i(k+4)$ th group, $i(k+5)$ th group, etc., until the last group of the $k$ th time interval is identified. According to the leading position and the extent of each group calculated in the manner described above, the number of the groups in tunnel of $i$-type vehicle at any moment $t$ can therefore be computed and is denoted as $I_{g i}(t)$. 
The partitioned traffic-flow data regarding a group of vehicle include the vehicular type, the number of vehicles, the averaged speed, the leading position, and the extent of each vehicular group. Besides, the number of group of each type of vehicle in tunnel at any interested moment can also be determined. With these deduced quantities, the resultant force exert on the tunnel air by all the vehicles in tunnel can be written as

$$
F_{1}(t)=\sum_{i=1}^{3} \sum_{j=k}^{k+I_{g_{i}}(t)} n_{i j}^{\text {in }} \frac{\rho}{2} C d_{i}\left(\bar{U}_{i j}-V\right)\left|\bar{U}_{i j}-V\right| A v_{i} \quad(k-1) \Delta t \leqslant t \leqslant k \Delta t .
$$

Based on the above quantities, one can calculate the traffic density distribution and the corresponding averaged speed of $i$-type vehicle along the tunnel at the moment $t$ with the following equations:

$$
D_{i}(t, x)=\sum_{j=k}^{k+I_{g_{i}}(t)} D_{i j}\left\{u\left[x-\left(x_{i j}-l_{i j}\right)\right]-u\left[x-x_{i j}\right]\right\} \quad(k-1) \Delta t \leqslant t \leqslant k \Delta t
$$

and

$$
\bar{U}_{i}(t, x)=\sum_{j=k}^{k+I_{g_{i}}(t)} \bar{U}_{i j}\left\{u\left[x-\left(x_{i j}-l_{i j}\right)\right]-u\left[x-x_{i j}\right]\right\} \quad(k-1) \Delta t \leqslant t \leqslant k \Delta t,
$$

where

$$
u[x-a]= \begin{cases}0, & x<a, \\ 1, & x>a,\end{cases}
$$

is a unit step function. Note that besides the traffic flow effect, the switch-on and -off of the jet fans are other factors influencing the wind speed in tunnel. This effect can be introduced into the theoretical model through $n_{\mathrm{F}}(t)$ in $F_{2}$, where $n_{\mathrm{F}}(t)$ is the number of fans under operation during the period $\left(t_{\mathrm{on}}<t<t_{\mathrm{off}}\right)$, while is 0 otherwise.

Eqs. (1)-(7) are to form the governing equation for the induced wind speed in road tunnel, which can be rewritten as

$$
\frac{\mathrm{d} V}{\mathrm{~d} t}=a(t) V^{2}+b(t) V+c(t) .
$$

The coefficients $a, b$ and $c$ are functions of the parameters regarding tunnel geometry, the tunnel flow, the mechanical ventilation system, the traffic, and so on. They can be expressed explicitly in terms of partition-group parameters as follows:

$$
\begin{aligned}
& a(t)=\left(\sum_{i=1}^{3} \sum_{j=k}^{k+I_{g i}(t)} n_{i j}^{\text {in }} \frac{\rho}{2} C d_{i} A v_{i} I_{i j}-f \frac{\rho}{2} \frac{L}{D_{\mathrm{h}}} A_{\mathrm{t}} I_{\tau}-K_{\text {port }} \frac{\rho}{2} A_{\mathrm{t}} I_{\text {port }}\right) /\left(\rho A_{\mathrm{t}} L\right) \\
& b(t)=\left(-\sum_{i=1}^{3} \sum_{j=k}^{k+I_{g i}(t)} n_{i j}^{\text {in }} \rho C d_{i} \bar{U}_{i j} A v_{i} I_{i j}-n_{\mathrm{F}} \rho A_{\mathrm{F}} V_{\mathrm{F}} K_{j} I_{\mathrm{F}}\right) /\left(\rho A_{\mathrm{t}} L\right) \\
& c(t)=\left(\sum_{i=1}^{3} \sum_{j=k}^{k+I_{g i}(t)} n_{i j}^{\text {in }} \frac{\rho}{2} C d_{i} \bar{U}_{i j}^{2} A v_{i} I_{i j}+n_{\mathrm{F}} \rho A_{\mathrm{F}} V_{\mathrm{F}}^{2} K_{j} I_{\mathrm{F}}+\left(P_{\mathrm{i}}-P_{\mathrm{e}}\right) A_{\mathrm{t}}\right) /\left(\rho A_{t} L\right) .
\end{aligned}
$$


where

$$
I_{i j}=\left\{\begin{array}{rl}
1 & \bar{U}_{i j}>V \\
-1 & \bar{U}_{i j}<V,
\end{array} \quad I_{\mathrm{F}}=\left\{\begin{array}{rl}
1 & V_{\mathrm{F}}>V \\
-1 & V_{\mathrm{F}}<V,
\end{array} \quad I_{\text {port }}=\left\{\begin{array}{rr}
1 & V>0 \\
-1 & V<0
\end{array}\right.\right.\right.
$$

Eq. (24) is a nonlinear first-order ordinary differential equation with variable coefficients, which together with the deduced traffic information can be numerically integrated from the initial wind speed $V(0)$ step by step to obtain the induced wind speed hereafter. An implicit central-difference scheme is employed to solve Eq. (24).

\subsection{An example of group-partition}

To show an example of the partitioned traffic flow in tunnel, we select the traffic data measured in the morning of $23 / 07 / 97$. This set of data includes the number of vehicles/10 s and averaged speed for each type of vehicles passing through the outlet portal, as shown respectively in Figs. 5 and 6. Every point in both figures accounts for the averaged traffic-flow data in every $10 \mathrm{~s}$, which is the smallest time interval can be set by the present data logger. Those points showing zero vehicular flow rate and zero-averaged speed represent that there was no vehicle passing through the outlet portal during the 10-s interval. From both figures we can see again that during these morning hours the traffic was dominated by the small-sized vehicles, the same as what is shown in Fig. 3. The data of Fig. 5 scatter widely than those of Fig. 3, which is because the former was evaluated in a much shorter time interval (every $10 \mathrm{~s}$ ) than the latter (every $5 \mathrm{~min}$ ). Fig. 6 shows that in most of the time the averaged speeds of three types of vehicles were all around $90 \mathrm{~km} / \mathrm{h}$, while at the rush-hour around $7: 45$ am the speeds all decreases to about $60 \mathrm{~km} / \mathrm{h}$, which is obviously due to the heavy traffic including a large number of large-sized vehicles. Note that although the large-sized vehicles were of a smaller amount, they afterall caused significant disturbances in the traffic flow.

A typical example resulted from the group-partition procedure is obtained by analyzing the traffic-flow data of Figs. 5 and 6 between $7: 00: 00$ (h:min:s) and $7: 01: 40$, and the result regarding the small-sized vehicle is shown in Fig. 7. In Fig. 7(a), it is seen that between $7: 00: 00$ and $7: 00: 10$ there were approximately eight groups of small-sized vehicle in the tunnel. The vehicles of these eight groups were of virtually uniform speed throughout the tunnel so that the group pattern of these groups did not change with time significantly. As one can see, the last group left tunnel at $7: 01: 15$, taking about $1 \mathrm{~min}$ and $10 \mathrm{~s}$ to pass through the Fu-De tunnel (1762 m long). This implies that the averaged speed of each group is approximately $90.6 \mathrm{~km} / \mathrm{h}$, being consistent with the data shown in Fig. 6. It is nevertheless possible to see from Fig. 7(b) that, although very little, the speed of each group was different from others. Due to these speed differences, overlaps between some groups occurred. In overlaps, the traffic densities are higher and appear as peaks in Fig. 7(a). Similar analysis applied for the traffic flow of the medium-sized and the large-sized vehicles. Because the traffic density of these two types was small as well as scattered, the partition of traffic was clearer and the overlaps between groups hardly appeared. 

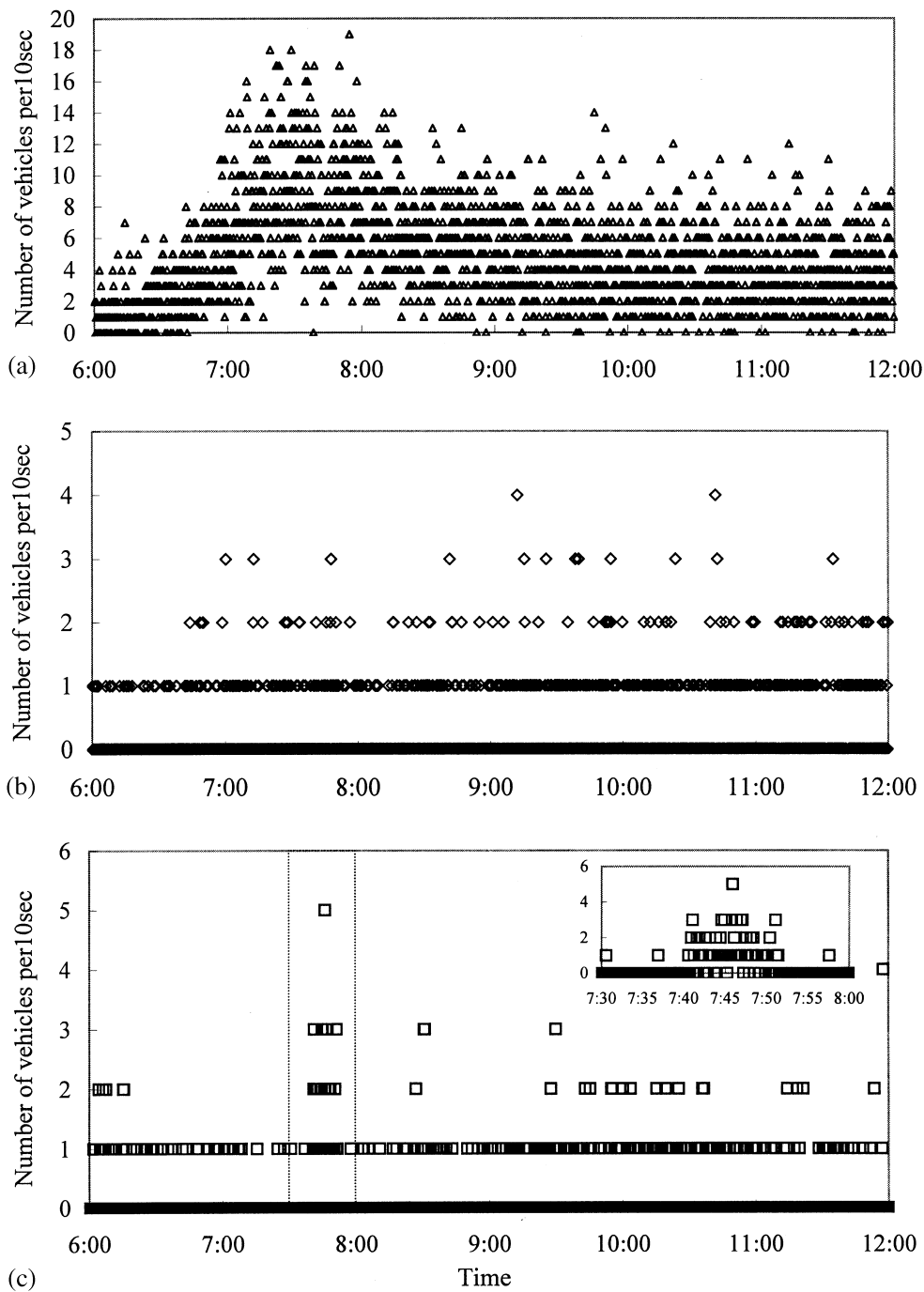

Fig. 5. The volume of traffic accounted for by the number of vehicles per $10 \mathrm{~s}$ for (a) small-sized vehicle (b) medium-sized vehicle and (c) large-sized vehicle. Each point represents the number of vehicles of the group passing the outlet portal in the 10-s interval. It is seen that the small-sized vehicle accounts for a major portion of the traffic and the traffic volume at the rush hour (between $7: 20$ to $8: 00 \mathrm{am}$ ) is more than twice than the other time. On the other hand, the medium- and the large-sized vehicles were of relatively small amount, except there happened to be a motorcade of large-sized vehicles passing the outlet portal between $7: 40$ and $7: 50$ (see enlarged figure on the upper-right corner), which reduced the averaged vehicular speed to about $60 \mathrm{~km} / \mathrm{hr}$ (see Fig. 6).

The group-partition procedure is applied to the whole period of interest $(6: 00: 00-12: 00: 00)$ and the deduced traffic information along the tunnel is provided for the theoretical ventilation model through Eq. (21) to calculate the induced 

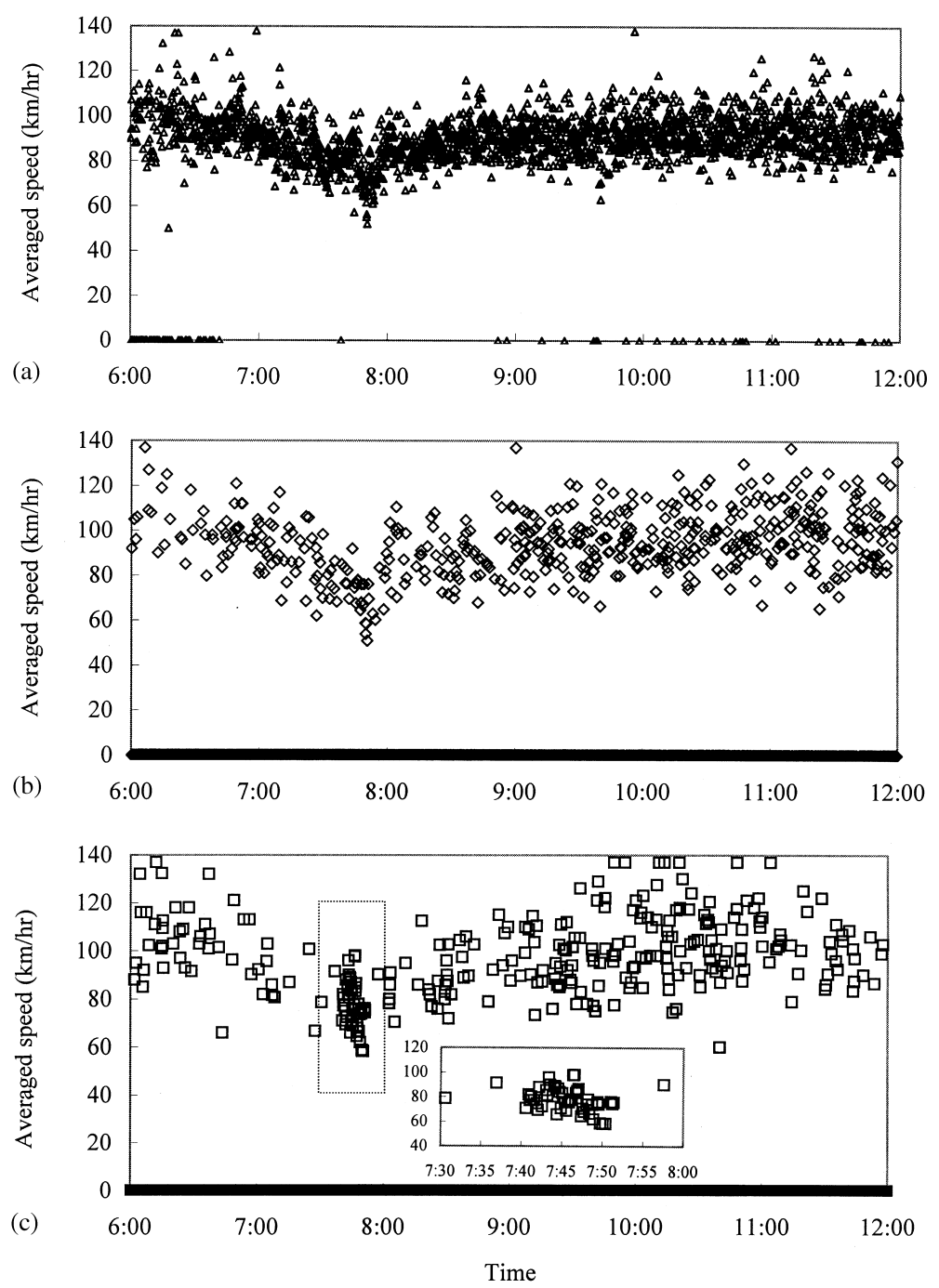

Fig. 6. The averaged speed of (a) small-sized vehicle, (b) medium-sized vehicle and (c) large-sized vehicle on 23/07/97 from $6: 00$ to $12: 00 \mathrm{am}$. Except at about $7: 45$ am when the motorway was suffering from a minor traffic jam caused by a motorcade of large-sized vehicles, the averaged speed was about $90 \mathrm{~km} / \mathrm{h}$, which is the speed limit of the motorway, reflecting a fact that the traffic in Fu-De tunnel was generally as smooth as designed. The enlarged figure shows that there were a motorcade of large-sized vehicle between $7: 40$ to $7: 50 \mathrm{am}$.

wind speed and the pressure distribution in tunnel. The reason that the vehicle groups are partitioned according to different size of vehicle is due to the fact that the drag coefficient of vehicle is size-dependent. The drag coefficient, as will be shown later, is of significant influence on the induced wind speed, especially the transient behavior of the induced wind to which we pay much attention in this study. In addition to the 


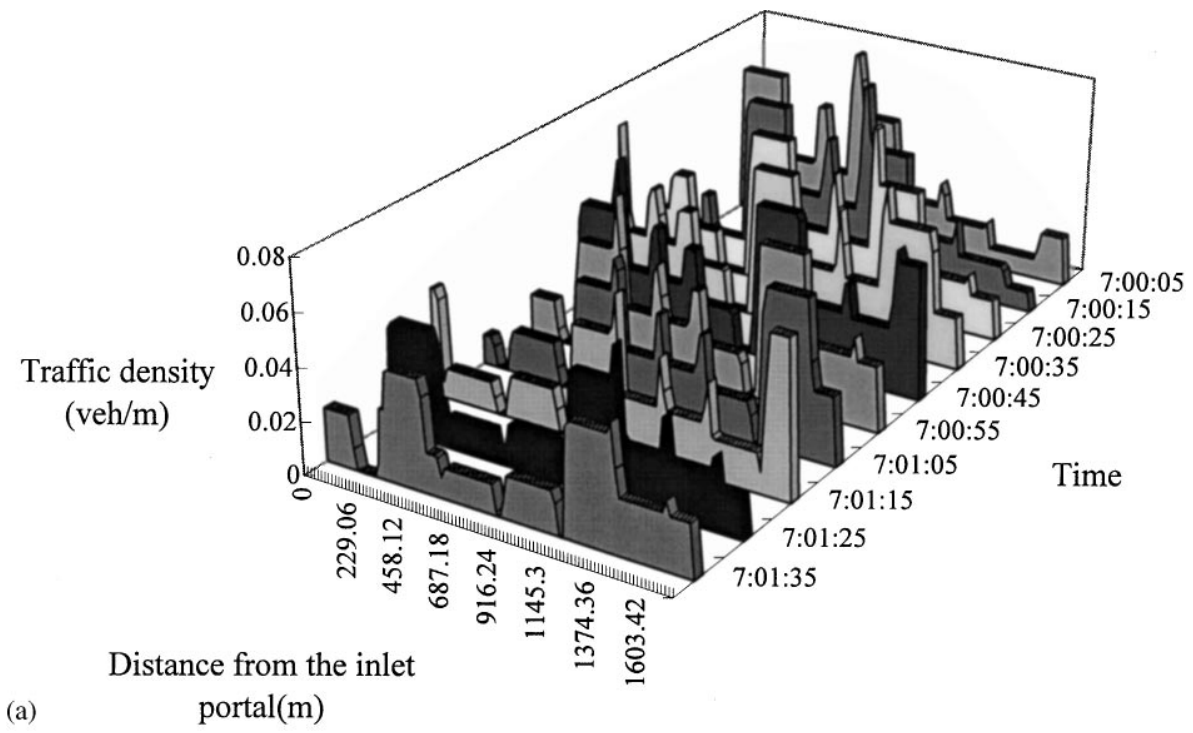

(a)

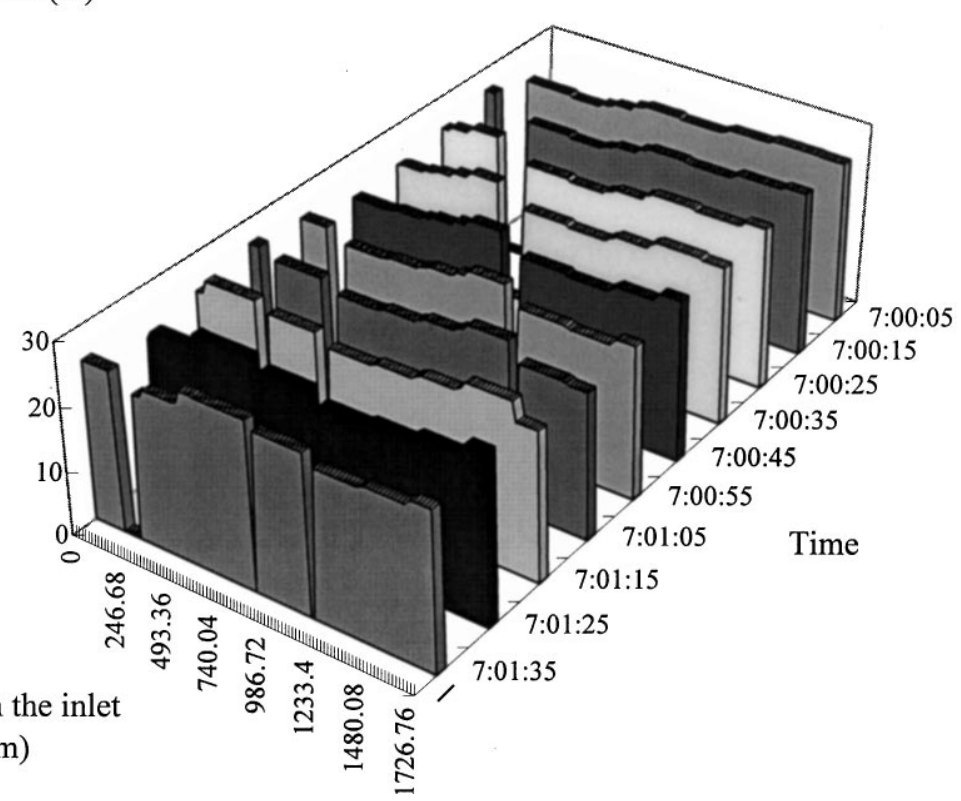

Fig. 7. Results obtained by the group-partition procedure for small-sized vehicles from $7: 00: 00$ to $7: 01: 40$ on 23/07/1997. (a) The traffic density in terms of number of vehicles per meter. The vehicles in tunnel are partitioned into several groups, all move with constant speeds. The peak between two neighboring groups indicates that there is overlap between them and the valley represents that there is no vehicle of the same kind in between. (b) The vehicle speed of each group. Each group is assumed to move constantly with its own speed. The speed differences between groups is small. 
Table 1

Values of parameters used in the present study [5]

\begin{tabular}{ll}
\hline$\rho$ & $1.16 \mathrm{~kg} / \mathrm{m}^{3}$ \\
$L$ & $1762 \mathrm{~m}$ \\
$D_{\mathrm{h}}$ & $10.38 \mathrm{~m}$ \\
$A_{\mathrm{t}}$ & $93.88 \mathrm{~m}^{2}$ \\
$f$ & 0.025 \\
$C_{\mathrm{d}}$ (small-sized vehicles) & $0.2^{\mathrm{a}}$ \\
$C_{\mathrm{d}}$ (medium- and large-sized vehicles) & $0.56^{\mathrm{a}}$ \\
$A v$ (small-sized vehicles) & $2.5 \mathrm{~m}^{2}$ \\
$A v$ (medium- and large-sized vehicles) & $7.11 \mathrm{~m}^{2}$ \\
$K_{j}$ & 0.4 \\
$A_{\mathrm{F}}$ & $2.01 \mathrm{~m}^{2}$ \\
$V_{\mathrm{F}}$ & $30 \mathrm{~m} / \mathrm{s}$ \\
$K_{\text {port }}$ & 0.6 \\
\hline
\end{tabular}

${ }^{a}$ The drag coefficients are lower than the values determined by single-vehicle experiments, which used to be in average about 0.3 for small-sized and 0.8 for medium- and large-sized vehicles. Because on the road the vehicles were covered by the wakes induced by other vehicles, in which irregular vortices prevail. The drags of the air imposing on the vehicles are therefore greatly reduced, say $30 \%$ [5]. The vaule of $K_{j}$ was determined from another analysis [5], in which we applied the unsteady one-dimensional model and employed relevant experimental data to seek the proper value of $K_{j}$ in $\mathrm{Fu}-\mathrm{De}$ tunnel.

drag coefficient of vehicle, there are other parameters being also influential on the induced wind speed, such as the friction factor of tunnel wall and the pressure-rise coefficient of jet fans. These parameters need to be determined before the analysis of the induced wind can be implemented. The latter two parameters affect only the averaged behavior of wind and the drag coefficient affects both the averaged and the transient behavior of the wind. The detail procedure for determining these parameters can be found in Ref. [5]. The values of the parameters used in the present analysis are listed in Table 1.

\section{Comparison between theoretical and experimental results}

Besides the traffic data, the in situ measurement system also provides the data of wind speed, pressure, and temperature of the air in tunnel at three locations, respectively, at 50,500, and $1000 \mathrm{~m}$ from the outlet portal. In this paper, the measured and theoretical wind speeds are used to compare each other and the computed pressure distributions along the tunnel are discussed .

We apply the theoretical model as well as the group-partition procedure on the traffic data measured in the morning of $23 / 07 / 97$, which have been shown in 


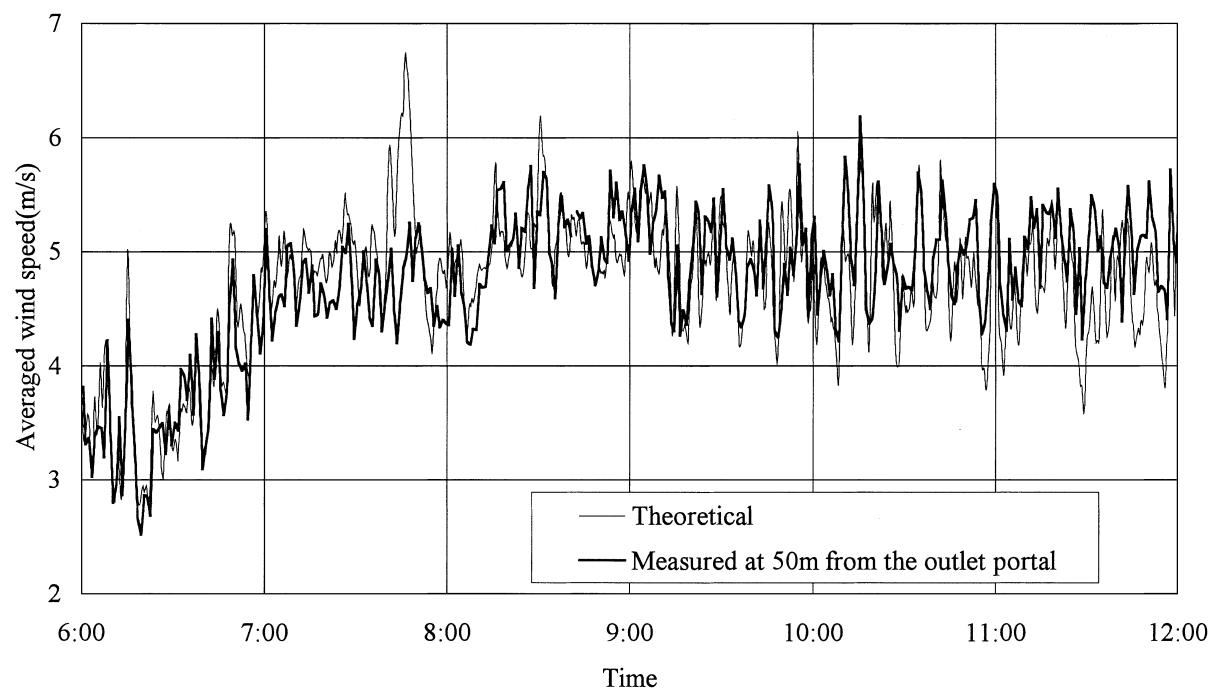

Fig. 8. A comparison between the wind speeds obtained by both theoretical and experimental approaches. The data were measured in the morning of 23/07/1997. Note that the comparison is generally very nice except at about $7: 45$ am when the traffic was heavy and the large-sized vehicles account for a significant portion of the traffic, suggesting that the drag forces of all the vehicles behind those large-sized trucks may be a little overestimated. Otherwise, the theoretical model based on the partitioned traffic groups might be able to more accurately predict the transient behavior of the induced wind speed.

Figs. 5 and 6. The computed-induced wind speed varying with time is shown in Fig. 8, in which the measured wind speed is also shown for the purpose of comparison. It is found that the comparison is very nice, especially that the transient behavior of the induced wind speed in terms of both the phase and the amplitude of variation are all in good agreement. We note nevertheless that at about 7:50 am when the number of truck increases dramatically, the computed results are larger than the measured data, suggesting that the drag coefficient of the vehicles behind those large trucks can be overestimated in this traffic situation. Namely, the drag coefficient of the vehicles shaded by the wake of trucks shall be smaller than that shown in Table 1. Otherwise, as small-sized vehicle dominates the traffic, the prediction from present theoretical approach can perfectly reflect the reality.

More evidence supporting the present theoretical approach can be obtained from other cases of different occasions. To confirm further the superiority of the present theoretical approach, we had examined a set of data measured in the evening of 27/07/97, which due to some unknown reasons were piecewise discontinuously recorded. Whereas the traffic data measured by the Autoscope were complete, so that we were able to apply the present theoretical approach to predict the induced wind speed in the whole period. Results (not shown in the present paper) showed that the theoretical results could essentially fill up the gaps between the discontinuous data, resulting in a complete picture of the variation of the induced wind in the tunnel. 


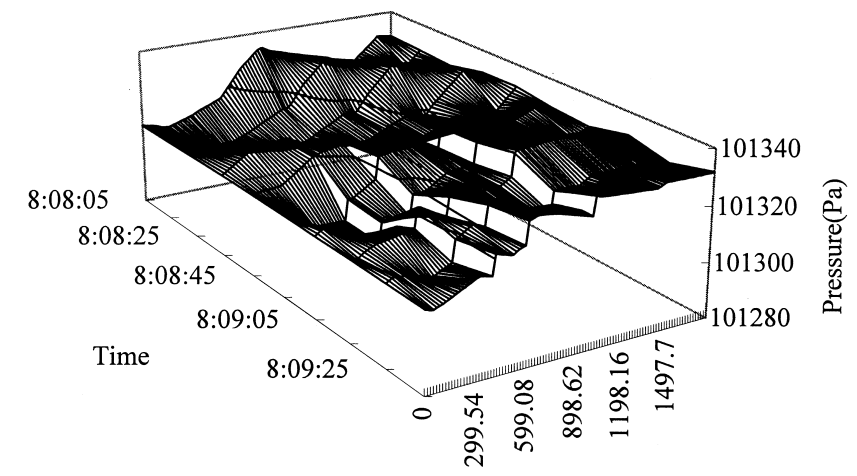

(a)

Distance from the inlet portal (m)

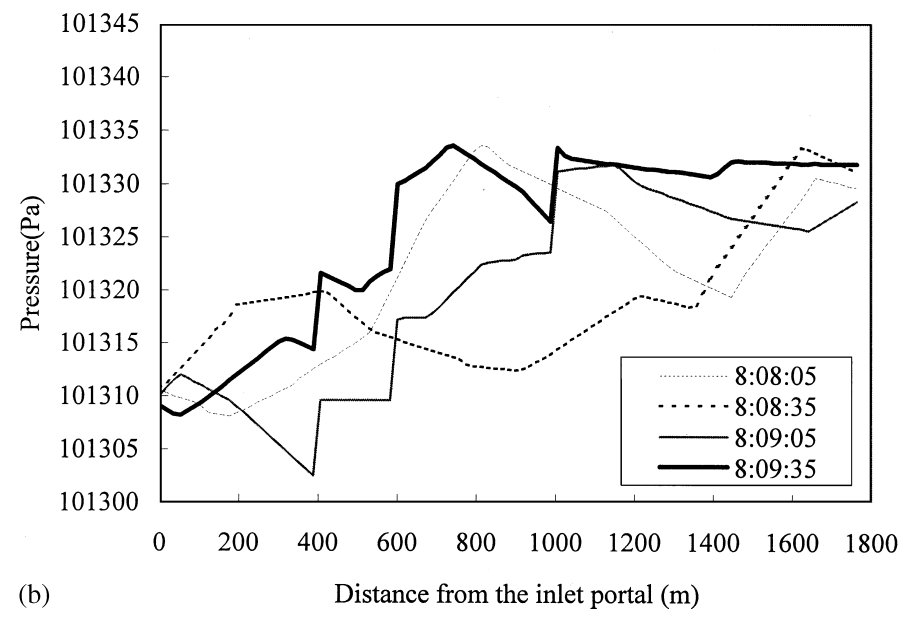

Fig. 9. The predicted pressure distribution along the tunnel. (a) The pressure distributions represented in time and space. (b) The pressure distributions at four different times. Note that there were three sudden jumps of pressure after $8: 08: 55$ in tunnel, which is due to the fact that the three jet-fans in tunnel were turned on at that time.

Because the pressure sensors we posed in tunnel were not of enough precision to detect the small fluctuation of pressure, the transient behavior of the pressure along the tunnel could not be detected by the measurement but can be predicted by the present theoretical approach. Based on the wind speed and the partitioned traffic flow along the tunnel, the pressure distribution along the tunnel can be obtained by numerically integrating the momentum equation from the inlet portal towards the outlet portal. The computed pressure distribution along the tunnel from $8: 08: 00$ to $8: 09: 40$ are shown in Fig. 9(a), and with a more precise scale the pressure distributions at four different times are shown in Fig. 9(b).

The reason to show the pressure distribution in this selected time period is simply because the three jet fans located, respectively, at 400, 600 and $1000 \mathrm{~m}$ from the inlet 

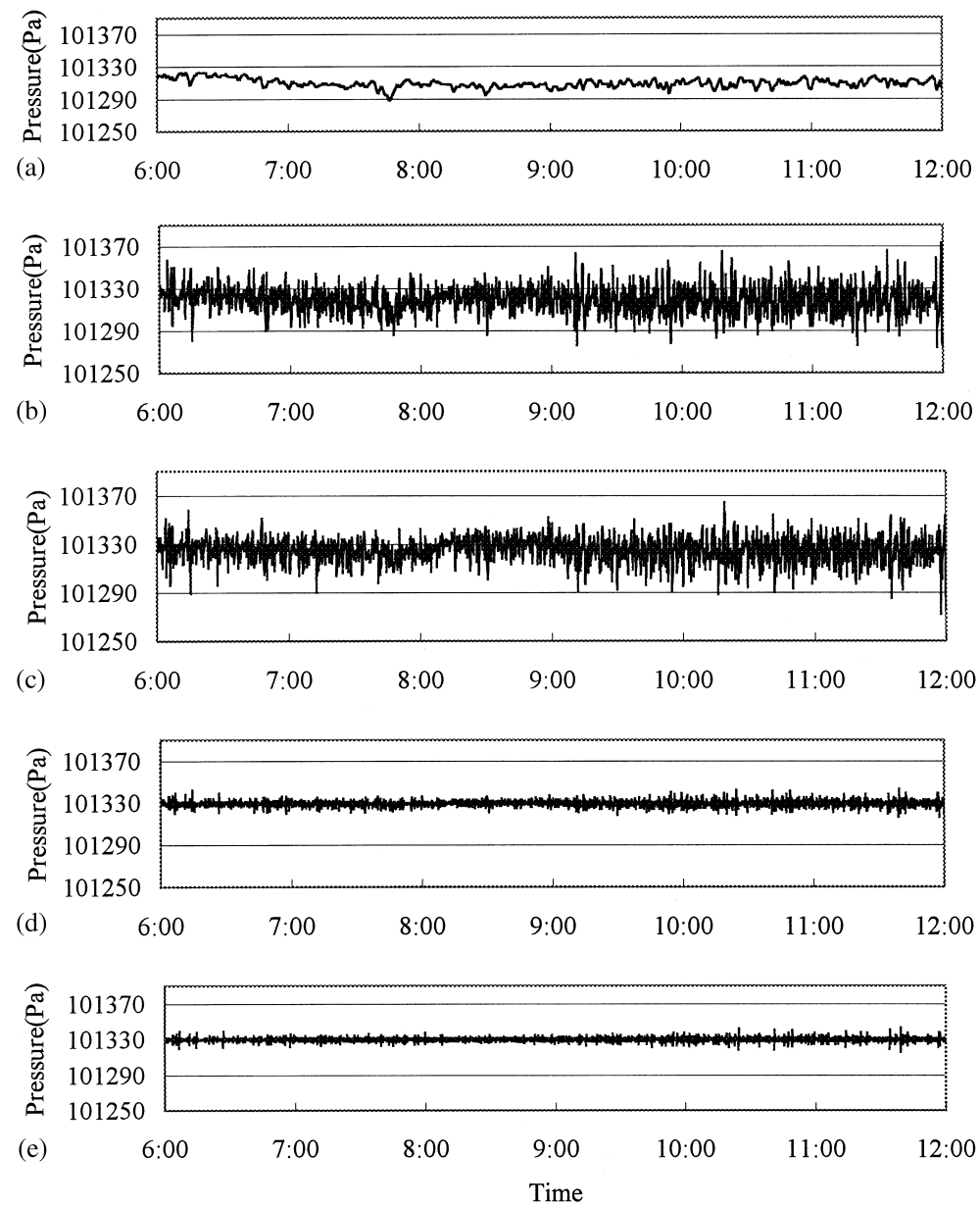

Fig. 10. Time evolutions of the pressure at five different locations in tunnel. (a) at the inlet portal. (b) at $1000 \mathrm{~m}$ from the outlet portal. (c) at $500 \mathrm{~m}$ from the outlet portal. (d) at $50 \mathrm{~m}$ from the outlet portal. (e) at the outlet portal. Fluctuation of pressure is more intense at the place farther from both the inlet and outlet portals, implying that the pressure fluctuation caused by the piston effect of vehicles is more intense within the tunnel while is more relax when close to the portals.

portal were turned on at $8: 09$ in the morning. So that the computed pressure shown in Fig. 9 can illustrate the effects imposed from both the jet fans as well as the traffic. Both Fig. 9(a) and (b) illustrate that before the jet fans were set into operation, the pressure was the lowest near the inlet portal, increased gradually along the tunnel due to the piston effect of vehicle motion, and eventually reached the atmosphere pressure at outlet portal. The pressure in most part of tunnel was below the atmosphere pressure, which was about $101330 \mathrm{~Pa}$. While as the three jet fans were set into operation, the lowest pressure occurred at the suction side of the first jet fan and the highest pressure appeared at the discharging side of the last jet fan. Each jet fan 
could increase the pressure by about $8 \mathrm{~Pa}$. We also show in Fig. 10 the computed pressures at five different locations in tunnel. Results show that the inlet portal pressure was always below the atmosphere pressure and the pressure at outlet portal was virtually equal to the atmosphere pressure. Fig. 10(b-d) reveal the important phenomena that the pressure is fluctuating more violently at deeper locations of tunnel.

\section{Conclusions}

We have proposed a novel approach with which the transient behavior of the induced wind speed in a motorway tunnel can be accurately predicted when the traffic data measured at the outlet portal are provided. This novel theoretical approach is set up on the basis of the so-called group-partition dilemma, which essentially partitions the vehicles into different groups, according to both the size of vehicle and the measured time intervals. The size of vehicle is categorized into three types, i.e., the large-size, the medium-size, and the small-size, and the time interval can be adjustable according to the specification of the data logger. To the present logger used to record the traffic data, a time interval can be as small as $10 \mathrm{~s}$. By substituting the partitioned traffic data into the one-dimensional force equation, the unsteady induced wind speed in tunnel can be accurately computed. The theoretical results have been shown to be in nice comparison with the measured data. Emphasis has been placed on the prediction of the transient state of the induced wind and the superiority of the present approach has been confirmed by the nice agreement in terms of the phase and amplitude of the fluctuation of induced wind speed between the theoretical and experimental results. The computed wind speed can then be employed to compute both the temporal variations of temperature and pressure along the tunnel, in which the computed temperature distribution along the tunnel agrees also nicely with the measured data. The present approach is believed to be also valid to predict the unsteady behavior of the variation of pollutant along the tunnel.

As far as the engineering application is concerned, the present theoretical approach may provide a scheme with which the monitoring of ventilation in tunnel can be greatly simplified. The present one-dimensional theoretical model can predict reasonably the induced wind speed as long as the traffic data at one point near tunnel, for example the outlet portal, are provided. Through this approach, the transient state of the ventilation in the tunnel can be well predicted when the traffic is smooth.

\section{Acknowledgements}

This work has been supported by the Taiwan Area National Expressway Engineering Bureau, Ministry of Transportation and Communications through grant 860NO73 and the National Science Council through grants NSC 87-2212-E-034-004, NSC-88-2212-E-034-002 and NSC-88-2212-E-002-030. 


\section{References}

[1] K.H. Huang, Transient analysis of the dispersion of vehicle pollution within a highway tunnel, Master Thesis, The University of Tennessee, Knoxville, Dec., 1980.

[2] A. Mizuno, T. Sasamoto, I. Aoki, The emergency control of ventilation for the trans-Tokyo Bay Tunnel, Proceedings of the 7th International Symposium on the Aerodynamics \& Ventilation of Vehicle Tunnels, 1991, pp. 365-384.

[3] P.F. Hartman, N.P. Costeris, L. Swart, Calculation method for longitudinal ventilation system, in: A. Glerum et al. (Ed.), Ventilation of Road Tunnels, Royal Institute of Engineers (KIVI), Division of Civil Engineering, 1991, pp. 55-76.

[4] H.M. Jang, On the modeling of road-tunnel ventilation systems, Hwa Kang J. Eng. 11 (1997).

[5] F. Chen, H.M. Jang, Theoretical and experimental studies of ventilation in road tunnel, Technical Report \#096-1, Taiwan Area National Expressway Engineering Bureau, Ministry of Transportation and Communications, Dec., 1997. 